\title{
Test re-test reliability and construct validity of the star-track test of manual dexterity
}

Niels Kildebro, Ilda Amirian, Ismail Ig Gögenur, Jacob Rosenberg

Objectives: We wished to determine test re-test reliability and construct validity of the star-track test of manual dexterity. Design: Test re-test reliability was examined in a controlled study. Construct validity was tested in a blinded randomized crossover study. Setting: The study was performed at a university hospital in Denmark. Participants: A total of 11 subjects for test re-test and 20 subjects for the construct validity study were included. All subjects were healthy volunteers. Intervention: The test re-test trial had two measurements with 2 days pause in between. The interventions in the construct validity study includedbaseline measurement, intervention 1: fatigue, intervention 2: stress, and intervention 3: fatigue and stress. There was a 2 day pause between each intervention.

Main outcome measure: An integrated measure of completion time and number of errors was used. Results: All participants completed the study (test re-test $n=11$; construct validity $n=20$ ). The test re-testshowed a strong Pearson product-moment correlation ( $r=0.90, n=11, P<0.01$ ) with no sign of learning effect. The 20 subjects in the construct validity trial were randomized to the order of the four interventions, so that all subjects completed each intervention once. A repeated measures ANOVA determined that mean integrated measure differed between interventions $(p=0.002)$. Post hoc tests using Bonferroni correction revealed that compared with baseline all interventions had significantly higher integrated scores ranging from $47-59 \%$ difference in mean.

Conclusion: The star track test of manual dexterity had a strong test re-test reliability, and was able to discriminate between a subject's normal manual dexterity and dexterity after exposure to fatigue and/or stress. 
2 Title:

3 Test re-test reliability and construct validity of the star-track test of manual dexterity.

4

5 Authors:

6 Niels Kildebro ${ }^{1}$, Ilda Amirian ${ }^{1}$, Ismail Gögenur ${ }^{2}$, Jacob Rosenberg ${ }^{1}$

7

8 From:

$9{ }^{1}$ Center for Perioperative Optimization, Department of Surgery, University of Copenhagen, Herlev

10 Hospital, Herlev Ringvej 75, 2730 Herlev, Denmark

$11{ }^{2}$ Køge Hospital, Department of Surgery, Lykkebækvej 1, 4600 Køge, Denmark

13 Corresponding author:

14 Niels Kildebro, E-mail: nielskildebro@gmail.com, Phone: +45 31103626

15

\section{Conflicts of interest}

17 None

18

19 


\section{Background}

更

A surgeon's manual dexterity is often an outcome parameter in studies examining environmental effects such as work environment or night shifts on surgeons (Dorion \& Darveau 2013; Amirian et al. 2014). Simulation tools are often used, but these are mostly time consuming tests that are not readily available. Often a study needs a tool that is easy to administer and is portable so that it can be used where the study calls for it. One such device was used in an interventional study for measuring surgeons' accuracy (Dorion \& Darveau 2013). The surgeons were to follow a star shaped track with a pair of surgical scissors and each time the scissors touched the border of the track, an error was counted. The track was to be completed 3 times and errors were noted. The study stated that it was examined for test re-test reliability. The authors reported a Pearson's correlation of $r=0.955$ (Dorion \& Darveau 2013; Savoie \& Prince 2002).

However, the test needs further validation if it is to be used in further research (Fess 1995). The method of examining the test re-test reliability of the test has not been described previously. The psychometric qualities have not been tested thoroughly enough to state that the test is valid and measures the intended characteristics (Fess 1995; Law 1987; Rudman \& Hannah 1998). Furthermore, the test has no equipment construction standards or instructions of use available, so the test lacks repeatability and reproducibility (Fess 1995; Law 1987; Rudman \& Hannah 1998;). It is necessary to further explore the reliability and validity of the test. With these parameters established, the test could be an excellent tool for measuring manual dexterity, and may be useful in future studies, providing an assessment tool that requires short time to be administered and is commercially available. application, test-retest reliability and construct validity for the star shaped test of manual dexterity.

\section{Method}


61 Instructions for administration of the test.

\section{Equipment construction standards} 2014)

T
with all its components is illustrated in picture 2 .

\section{Instructions for administration of the test.}

The star-track test of manual dexterity consists of the following components:

- Replacement star Model 32532A from Lafayette instruments (lafayette instruments

○ MakeyMakey from joylabz.com (JoyLabz 2014).

○ Computer running the software Star Track 32bit.exe (Nørregaard 2014).

- Standard Metzenbaum surgical scissors

The replacement star Model 32532A from Lafayette instruments is a metal plate measuring $22 \mathrm{~cm} \times 22$

$\mathrm{cm}$ with a star shaped track in its center. The six-pointed star shaped track measures $15.3 \mathrm{~cm}$ from

point to opposite point. The track is $0.9 \mathrm{~cm}$ wide and is made of a non-conducting material. A

MakeyMakey is an inventor kit that can turn everyday objects into touchpads and combine them with a computer. This is explained further at http://makeymakey.com. The MakeyMakey is used to connect the metal plate and the Metzenbaum surgical scissors to a computer. In picture 1, detailed measurements of the dimensions of the metal star shaped track are shown. A complete setup of the test

The test is set up in the following way. The program Star Track 32bit.exe needs to be installed on the computer used for the test. It is recommended to use a laptop to increase the transportability of the test.

Star Track 32bit.exe is a freeware that can be downloaded and installed from

65 http://bitbucket.org/lassebn/star-track. MakeyMakey is used to connect the components of the star-

66 track test using the following steps: 1: Connect the MakeyMakey to the computer via a USB cable. The 67 MakeyMakey will auto install (JoyLabz 2014). 2: Connect the "space" part of the MakeyMakey to the 
68 Metzenbaum surgical scissors using two alligator clips linked together. 3: Connect the "ground" of the

69 MakeyMakey to the side of the metal plate using an alligator clip.

The test should be performed in a quiet room without distractions. The examiner places

71 the metal plate about $10 \mathrm{~cm}$ from the edge of a table where the subject is sitting comfortably. The

72 examiner instructs the subject to use the scissors in the hand that he wishes to examine. Using the

73 scissors, the subject must follow the star shaped track ten times, five times clockwise and five times

74 counterclockwise. All ten rounds are completed continuously. The tip of the scissors must be in contact

75 with the star shaped track during the entire test. Each time the scissors come into contact with the

76 border of the track, an error is registered. Completion time and number of errors are registered

77 automatically by Star Track 32bit. The examiner should read the following instructions to the subject:

78 "To complete the test, you must follow the star shaped track with the surgical scissors. You are to

79 complete ten rounds, five rounds clockwise and five rounds counterclockwise. All ten rounds are to be

80 completed continuously. You are to complete the ten rounds as quickly as possible with as few errors

81 as possible. An error is counted every time the scissors touch the border of the star shaped track. The

82 scissors must touch the plate at all times during the test. "

83 With Star Track 32 bit running on the computer, the examiner names the test result file. When he/she

84 presses the enter button, the test will begin. When the subject completes the final round, the examiner

85 presses " $\mathrm{q}$ " to stop the test.

87 Scoring

88 The Star Track 32bit program automatically records the time (in seconds) it takes to complete the test.

89 It also automatically records errors.

91 Construct validity 
93 We wished to study whether the star-track test would be able to distinguish between the base level of

94 manual dexterity of a person and the level of manual dexterity when the person was fatigued and/or

95 stressed. This was done by conducting a randomized crossover study. Each subject was to complete the star-track test four times. At each trial they were randomly assigned to different interventions. Each subject was to complete all four interventions, and never the same intervention more than once. Each trial was separated by two days' pause. The interventions were: Baseline measurement, the subject completed the star-track test without further intervention. Intervention 1: The subject was fatigued in his dominant arm before completing the star-track test. Intervention 2: the subject was stressed while performing the star-track test. Intervention 3: The subject was fatigued prior to the star-track test and stressed while performing the star-track test. Completion time and number of errors in the star-track test were measured at all four interventions using the Star Track 32bit software. The order in which each subject received the four interventions was randomized (http://www.randomization.com). Using the randomization list, a research fellow, who was not involved in the study, packed and sealed 4 opaque envelopes (labeled day 1 , day 4 , day 7 and day 10) containing the randomization labels for each subject. These envelopes were opened on the respective days just before the test commenced, so that the subject and examiner were blinded until that point.

\section{$\underline{\text { Subjects for construct validity }}$}

We aimed to include 20 subjects. The subjects were all volunteers and gave written informed consent before inclusion. They were recruited from local universities, dormitories and hospitals by advertising with posters. Subjects had to understand Danish (written and spoken). They were excluded if they were diagnosed with heart, endocrine, neurological, autoimmune or psychological disease, suffered from 
115 sleep disorders or had muscular-skeletal disorders of the upper extremities (e.g. osteoatrosis, rotator 116 cuff syndrome, hand injuries).

118 Method of achieving muscular fatigue

119 The fatigue was achieved by letting the subject hold a $2.5 \mathrm{~kg}$ weight in the dominant hand, and holding

120 the dominant arm to 90 degrees flexion. They were to hold this position without moving for as long as

121 possible. The subjects then proceeded to complete the star-track test within 10 seconds. This test has

122 previously been used to measure muscular fatigue (Dorion \& Darveau 2013) and is described in

123 occupational health literature as a way to achieve static muscular fatigue (Chaffin 1973).

124

125 Method of inducing stress

126 The brain can focus on performing a specific task at normal level, as long as the mental resources

127 exceed the demand of the task in progress. If multiple tasks are to be performed at the same time, the

128 demands of the tasks will at some point exceed the mental workload tolerance. This will cause stress

129 and subjects will begin making errors (Boles \& Law 1998; Grier et al. 2008). According to the theory

130 of multiple resources, there are several mental resource pools, enabling several actions to be performed

131 simultaneously. However, if the actions performed require resources form the same pool, they will

132 cause stress more quickly (Wickens 2008). This allows for prediction of workload overload by

133 determining difficulty of the tasks undertaken and task interference. The star-track test is visually

134 perceived, requires spatial understanding and a manual response. The distraction was designed to drain

135 from these mental resources.

While the subject was performing the star-track test, the examiner would show the subject

13710 cards from a regular deck of cards. One card per round completed in the star-track test. The card

138 was placed near the metal plate of the star-track test, allowing the subject to have both the star-track 
139

140

141

142

143

144

145

146

147

148

149

150

151

152

153

154

155

156

157

158

159

160

161

162

and the card in his field of vision. The subject had to identify the card by rank and suit while performing the star-track test. According to the computational 3-D+1 model of multiple resources

(Wickens 2008), the difficulty of the tasks are both simple (following the star-track and identifying cards). The tasks share demands of workloads at two levels (perception and cognition). This gives a total interference of 4 (on a scale of interference from 0-8) (Wickens 2008). If the star-track test is able to detect this workload overload, a higher integrated score (longer completion time and/or more errors), compared with baseline should be scored while completing the test with distraction.

\section{Test re-test reliability}

The reliability was tested with a controlled design. The purpose of this test was to determine the testretest effect and whether or not the test was consistent over time. The subjects completed the star-track test with an interval of two days between tests. The test conditions were the same on each day of the trial. This design has been used previously to perform test-retest trials of manual dexterity (Aaron \& Jansen 2003). Completion time and errors were measured at both tests using the Star Track 32 bit software. To measure face-validity each subject was asked if he understood the purpose of the startrack test, and what they believed it was supposed to measure. We aimed to include 11 subjects. The method of recruitment, as well as inclusion and exclusion criteria were the same as for the validity test.

\section{$\underline{\text { Ethics and permissions }}$}

The study was registered at Clinicaltrials.gov (NCT02146443). The data collection was approved by the Danish Data Protection Agency (journal no: HEH-2014-060, I-Suite no. 02972). The study was exempt from approval by The Regional Danish Committee on Biomedical Research Ethics (protocol no: H-6-2014-031). All subjects volunteered and gave written informed consent prior to their participation. No compensation was offered for participating in the study. 
164 Statistics

165 All statistics were calculated using IBM SPSS Statistics version 22 (SPSS, Chicago, IL, USA) and

166 Microsoft Office Excel 2007. To receive a complete estimate of a subject's manual dexterity, we used

167 an integrated measure for completion time and number of errors (Silverman et al. 1993). The total

168 number of ranks for conducted trials were found ( 80 for validity; 22 for the test-retest) and mean rank

169 was calculated. The difference of completion time and number of errors from respective mean ranks

170 was calculated as a percentage in difference, and added on a per-subject basis to form an integrated

171 measure (Silverman et al. 1993). Since this was a pilot test, no sample size was calculated as no data

172 were available. Thus, sample size was determined by means of qualified estimate (Hertzog 2008).

173 Study population age was described as median (range). We used the Shapiro-Wilk test of normality to

174 determine that data were normally distributed. Mauchly's Test was used to test for Sphericity. We used

175 repeated measures ANOVA with post hoc testing with Bonferoni correction for intergroup

176 measurements in the validation study. The Bonferroni corrected p-value was calculated multiplying the

177 least significant differences by the total number of tests possible. Repeated measures ANOVA was

178 selected because the dependent variable (integrated measure of error and time) was measured on the

179 same group of people using different independent variables (interventions). Pearson correlation

180 coefficient was used for test re-test reliability analysis. Test days were also compared with paired

181 samples t-tests. $\mathrm{P} \leq 0.05$ was regarded as statistically significant.

182

183 Results

184 Construct validity 
185 A total of 20 subjects completed this study, 9 females and 11 males, with a median age of 26 years 186 (range 22-29). Of the participants 3 were left-handed and 17 were right-handed. The subjects were 187 students $(n=15)$, doctors $(n=2)$, nurses $(n=1)$ and engineer $(n=1)$. The integrated measures scores for 188 each of the four test arms of the crossover study can be found in table 1 . We tested for normality using

189 the Shapiro-Wilk test. It showed that the data for all four test arms of the crossover study did not 190 violate the assumption of normality (baseline $\mathrm{p}=0.73$; intervention $1 \mathrm{p}=0.67$; intervention $2 \mathrm{p}=0.79$; 191 intervention $3 \mathrm{p}=0.44)$. A repeated measures ANOVA was done to determine if the integrated 192 measures significantly differed from each other. Mauchly's Test of Sphericity indicated that the 193 assumption of sphericity of the data had not been violated $(\chi 2(5)=7.01, p=0.22)$ and thus no 194 correction was used in the repeated measures ANOVA. It was determined that mean integrated 195 measure differed significantly between interventions $(\mathrm{p}=0.002)$. Post hoc tests using Bonferroni 196 correction revealed that compared with baseline all interventions had significantly higher integrated 197 scores, indicating that the test was able to differentiate between the baseline and the interventions (see 198 table 2). Furthermore; intervention 3 scored higher integrated measure than intervention 1 and 2, with a 199 mean difference in integrated measure of 0.12 and 0.10 respectively (see table 1 ), although this 200 difference was statistically insignificant $(\mathrm{P}=1$ for both).

202 Test re-test reliability:

203 A total of 11 subjects completed this study, hereof 5 females. The median age was 27 years (range 22204 35). Two of the subjects were left-handed and nine were right-handed. The subjects included 7 205 students, 1 engineer and 3 nurses. The integrated measures scores for each test day are presented in 206 207 days. The data showed no violation of normality (Shapiro-Wilk test of test day $1 \mathrm{p}=0.32$ and test day 
$2084 \mathrm{p}=0.25$ ), linearity or homoscedasticity. There was a strong, positive correlation between the 209 integrated measures of the two test days $(\mathrm{r}=0.90, \mathrm{n}=11, \mathrm{P}<0.01)$.

Test day 1 and test day 4 were compared with paired samples t-tests to ensure that the

211 Pearson correlation coefficient was not high due to a consistent difference (e.g. learning effect). There

212 was no significant difference in integrated measure $(\mathrm{p}=0.21)$.

\section{Discussion}

215 The star-track test was able to detect a difference between the baseline measurement and all three of

216 the interventions in the construct validity study. This indicates that the test was able to discriminate

217 between a person's baseline and impaired manual dexterity due to fatigue and/or stress. The test re-test

218 reliability showed that the star-track test had a strong test re-test reliability.

The purpose of the star-track test was to be an evaluative tool for measuring manual

220 dexterity, and to measure changes in individuals. More specifically, the target population of the test

221 was subjects with no impairment or disease in the upper extremities. The test involved a surgical

222 instrument, it was meant to be used in future research to evaluate the manual dexterity of surgeons. If

223 the test is to be used as a descriptive tool, more studies should be conducted where normative data

224 should be collected on different groups of subjects standardized for age, gender, surgical experience

225 and maybe various impairments of the upper extremities. The star-track test has no predictive value

226 yet. To gain this, studies where surgeons' integrated measures in the star-track test are compared to 227 patient outcomes would be needed.

The reliability of the star-track test of manual dexterity has been explored in previous

studies (Dorion \& Darveau 2013; Savoie \& Prince 2002). They reported a Pearsons correlationwithr $=$

230 0.955. In this article, we confirmed the previous findings of a strong test-retest reliability of the star

231 track test, as our finding of $r=0.90$ was consistent with the previous research. Also, the trials ruled out 
232 any significant learning effects, which is especially important for the consistency of the test.

233 Furthermore, we described the method of obtaining the reliability results in detail, which had not been

234 done before. To make the star-track test accessible, equipment construction standards were provided

235 along with instructions for administration. We used components for construction of the test that were

236 commercially available, so that the test can be reconstructed and reproduced. This further established

237 the consistency and reliability of the test. As the data of the test were gained by means of a computer

238 program, we did not examine for inter-rater and intra-rater reliability, as the standardized computer

239 program minimized these factors.

All subjects easily understood that the test measured manual dexterity, which indicated

241 that the test had good face validity and was easy to understand.

The content validity had already been established, as the test was used to test the accuracy

243 of surgeons in a previous study (Dorion \& Darveau 2013). However, manual dexterity is a more

244 complete measure of a surgeon's skill than accuracy. Dexterity is the ability to manipulate objects with

245 your hands with a specific purpose in mind (Dunn et al 199; Baum \& Edwards 1995). Dexterity can be

246 subdivided into a static phase, and a dynamic phase which involves powergrip (adapting hand strength)

247 and precision handling of handheld objects (Kamakura et al. 1980). The characteristics of manual

248 dexterity are accuracy and speed (Aaron \& Jansen 2003). All this is needed in instrument handling and

249 therefore important to surgeons' technical skills (Memon et al. 2010). In this study we expanded the

250 measurement to be a more complete concept of manual dexterity by using an integrated measure of

251 errors and completion time. We believe that by doing this we have increased the content validity of the 252 star-track test.

Construct validity was explored in this study. The trend of the data showed that the more

254 stressed and/or fatigued a subject was, the higher the integrated measure of manual dexterity. This

255 trend is illustrated in figure 1. It detected a mean difference of $10-12 \%$ between intervention 3 and both 
256 intervention 1 and intervention 2 . This indicated that the test might be able to measure different 257 intensities of stress and the effect of fatigue on the subjects' manual dexterity. However, the difference 258 was not statistically significant, and therefore we were unable to confirm this trend. Further testing 259 with a similar setup and a larger sample size should be conducted to further investigate this. The criterion validity of the test still needs to be established. This could be done in future 261 studies comparing data from the star-track test to other established accuracy tests and tests of manual 262 dexterity.

With the data presented in this article, we believe that the star-track test of manual

264 dexterity may be used in future research when testing the accuracy and manual dexterity of surgeons.

265 The star-track test can be used to discriminate between a subject's normal manual dexterity and after 266 exposure to fatigue and/or stress. 


\section{References}

Aaron DH, Jansen CW. 2003. Development of the Functional Dexterity Test (FDT): construction, validity, reliability, and normative data. Journal of Hand Therapy 16:12-21.

Amirian I, Andersen LT, Rosenberg J, Gögenur I. 2014. Laparoscopic Skills and Cognitive Function are not Affected in Surgeons During a Night Shift. Journal of Surgical Education 71:543-550.

Baum C, Edwards D. 1995. Position paper: occupational performance: occupational therapy's definition of function. American Occupational Therapy Association. American Journal of Occupational Therapy 49:1019-1020.

Boles DB, Law MB. 1998. A simultaneous task comparison of differentiated and undifferentiated hemispheric resource theories. Journal of Experimental Psychology: HumanPerception and Performance 24:204-215.

Chaffin DB. 1973. Localized muscle fatigue--definiton and measurement. Journal of Occupational Medicine 15:346-354.

Dorion D, Darveau S. 2013. Do micropauses prevent surgeon's fatigue and loss of accuracy associated with prolonged surgery? An experimental prospective study. Annals of Surgery 257:256- 259 .

Dunn W, Hinojosa J, Schell B, Thumsun LK, henfeljer SD. 1994. Uniform terminology for occupational therapy--third edition.American Occupational Therapy Association.American Journal of Occupational Therapy 48:1047-1054.

Fess EE. 1995. Guidelines for evaluating assessment instruments. Journal of Hand Therapy 8:144-148.

Grier R, Wickens C, Kaber D, Strayer D, Boehm-Davis D, Trafton JG, John MS. 2008. The red-line of workload: Theory, research, and design. Proceedings of the Human Factors and Ergonomics Society Annual Meeting: Sage Publications, p 1204-1208.

Hertzog MA. 2008. Considerations in determining sample size for pilot studies. Research in 

Nursing and Health 31:180-191.

293 JoyLabz. 2014. Makeymakey. Available at http://makeymakey.com(accessed 19 March 2014).

294 Kamakura N, Matsuo M, Ishii H, Mitsuboshi F, Miura Y. 1980. Patterns of static prehension in normal hands. American Journal of Occupational Therapy 34:437-445.

296 Law M. 1987. Measurement in occupational therapy: Scientific criteria for evaluation. Canadian Journal of Occupational Therapy 54:133-138.

298 Lafayette Instrument Company. 2014. Replacement Star Model 32532A. Available at http://www.lafayetteevaluation.com/product_detail.asp?itemid=216 (accessed 25 June 2014).

300 Memon MA, Brigden D, Subramanya MS, Memon B. 2010. Assessing the surgeon's technical skills: analysis of the available tools. Academic Medicine 85:869-880.

302

303
Nørregaard L. 2014. Star Track 32bit.exe. Daybuilder Solutions.Available at http://bitbucket.org/lassebn/star-track(accessed 5 December 2014).

Rudman D, Hannah S. 1998. An instrument evaluation framework: description and application to assessments of hand function. Journal of Hand Therapy 11:266-277.

Savoie S TR, Prince F. 2002. Hauteur de la table d'operation et performance chirurgicale. Thesis. Universite de Sherbrooke (Canada).

Silverman DG, O'Connor TZ, Brull SJ. 1993. Integrated assessment of pain scores and rescue morphine use during studies of analgesic efficacy. Anesthesia and Analgesia 77:168-170.

Wickens CD. 2008. Multiple resources and mental workload. Human Factors 50:449-455. 


\section{Legends}

312 Picture 1: Detailed measurements and dimensions of the metal plate with the star shaped track.

313 Picture 2: A complete setup of the test with all its components.

314 Table 1: Mean Integrated measures of validity test. Integrated measure of time and error during

315 completion of the star-track test of manual dexterity during each of the four test arms.

316 Table 2: Post hoc tests of repeated measures ANOVA. Integrated measure of time and error during

317 completion of the star-track test of manual dexterity, baseline compared to the three interventions.

318 Table 3: Mean integrated measures of test re-test trial. Integrated measure of time and error during 319 completion of the star-track test of manual dexterity.

320 Figure 1: The mean integrated measures of time and error during completion of the star-track test in 321 the construct validation study. 


\section{1}

The test setup

Picture 1 shows detailed measurements and dimensions of the metal plate with the star shaped track. Picture 2 shows a complete setup of the test with all its components. A) Shows the star shaped track used in the test. B) shows the The MakeyMakey. C) Shows the standard Metzenbaum surgical scissors and D) shows the computer running the software Star Track 32 bit.exe.

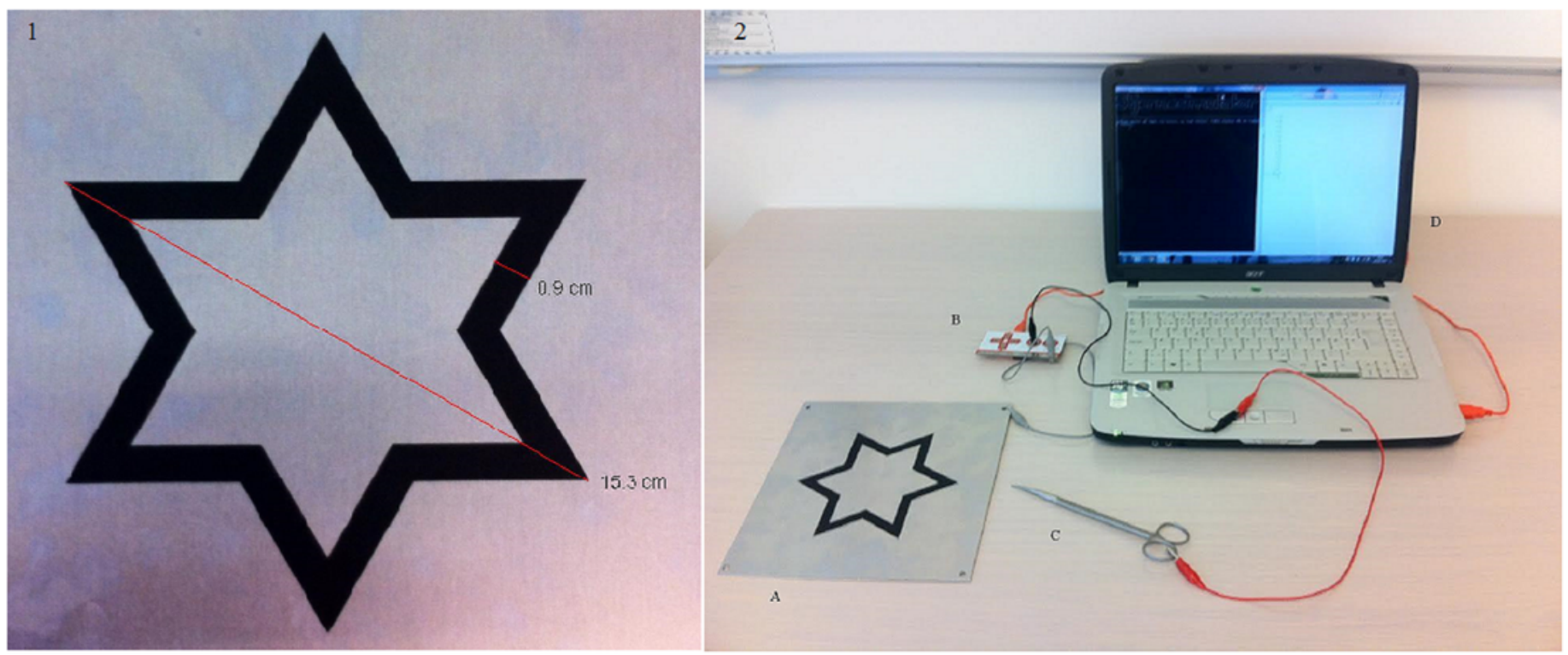


Table $\mathbf{1}$ (on next page)

Mean integrated measures of validity test.

Table 1: Mean integrated measures of validity test. Integrated measure of time and error during completion of the star-track test of manual dexterity during each of the four test arms. 


\section{PeerJ Reviewing Manuscript}

Table 1

\begin{tabular}{|l|l|l|l|}
\hline Test arm & $\mathrm{N}$ & Integrated measure & $\mathrm{SD}$ \\
\hline Baseline & 20 & -0.39 & 0.51 \\
\hline Intervention 1 & 20 & 0.08 & 0.61 \\
\hline Intervention 2 & 20 & 0.10 & 0.39 \\
\hline Intervention 3 & 20 & 0.20 & 0.55 \\
\hline
\end{tabular}


Table 2 (on next page)

Post hoc tests of repeated measures ANOVA.

Table 2:Post hoc tests of repeated measures ANOVA. Integrated measure of time and error during completion of the star-track test of manual dexterity. Baseline compared to the three interventions. 


\section{PeerJ Reviewing Manuscript}

Table 2

\begin{tabular}{|l|l|l|}
\hline Comparison & Mean difference & $p$ - values \\
\hline Baseline - intervention 1 & $-0.47(-0.93 ;-0.02)$ & 0.04 \\
\hline Baseline - intervention 2 & $-0.49(-0.92 ;-0.07)$ & 0.02 \\
\hline Baseline - Intervention 3 & $-0.59(-1.09 ;-0.09)$ & 0.01 \\
\hline
\end{tabular}

Values are presented as mean difference in integrated measure with $95 \%$ confidence interval. $p$ values calculated with post hoc tests using the Bonferroni correction. 
Table 3(on next page)

Mean integrated measures of test re-test trial.

Table 3: Mean integrated measures of test re-test trial. Integrated measure of time and error during completion of the star-track test of manual dexterity. 


\section{PeerJ Reviewing Manuscript}

\section{Table 3}

\begin{tabular}{|l|l|l|l|}
\hline Test re-test day & $\mathrm{N}$ & Mean integrated measure & $\mathrm{SD}$ \\
\hline Test day 1 & 11 & 0.07 & 0.62 \\
\hline Test day 2 & 11 & -0.05 & 0.72 \\
\hline
\end{tabular}


2

The mean integrated measures of time and error during completion of the star-track test in the construct validation study.

Figure 1: Integrated measure is percent from mean integrated measure of study population. Whiskers represent standard deviation. A positive score is a poorer than average performance (e.g. longer completion time and/or more errors) when compared to the mean score, while a negative score is better than average.

Figure 1

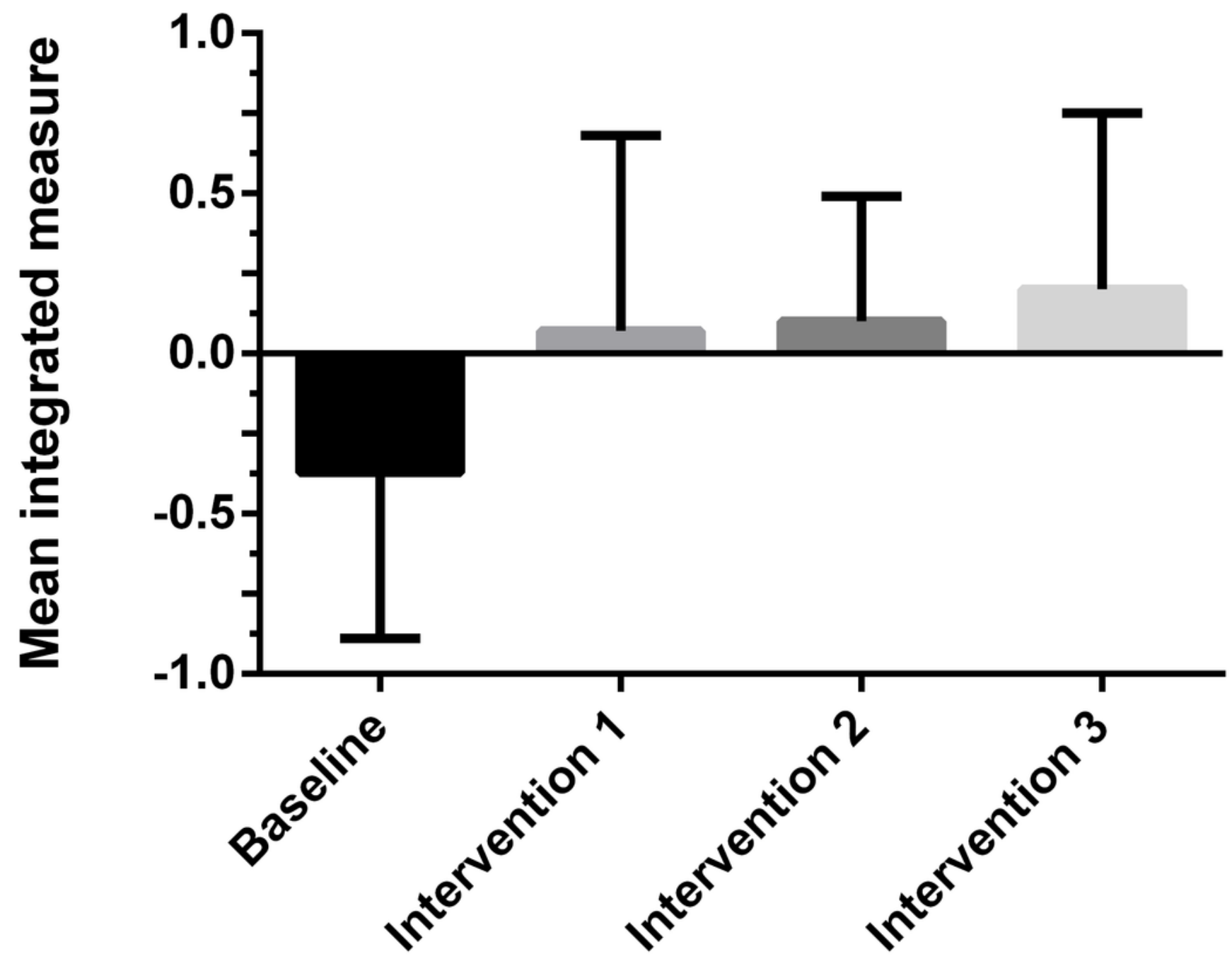

